\title{
Experimental and theoretical analysis for total electron scattering cross sections of benzene
}

Cite as: J. Chem. Phys. 151, 084310 (2019); https://doi.org/10.1063/1.5116076

Submitted: 21 June 2019 . Accepted: 07 August 2019 . Published Online: 30 August 2019

F. Costa, L. Álvarez, A. I. Lozano, F. Blanco (D), J. C. Oller (D), A. Muñoz, A. Souza Barbosa (D) M. H. F. Bettega (D), F. Ferreira da Silva (D), P. Limão-Vieira (D), R. D. White, M. J. Brunger (D), and C. García (D)

\section{ARTICLES YOU MAY BE INTERESTED IN}

Total cross section measurements for electron scattering from dichloromethane

The Journal of Chemical Physics 149, 244304 (2018); https://doi.org/10.1063/1.5080636

Revisiting the photoabsorption spectrum of $\mathrm{NH}_{3}$ in the 5.4-10.8 eV energy region

The Journal of Chemical Physics 151, 184302 (2019); https://doi.org/10.1063/1.5128051

Total electron scattering cross sections from thiophene for the (1-300 eV) impact energy range

The Journal of Chemical Physics 149, 134303 (2018); https://doi.org/10.1063/1.5050349

Lock-in Amplifiers Find out more today

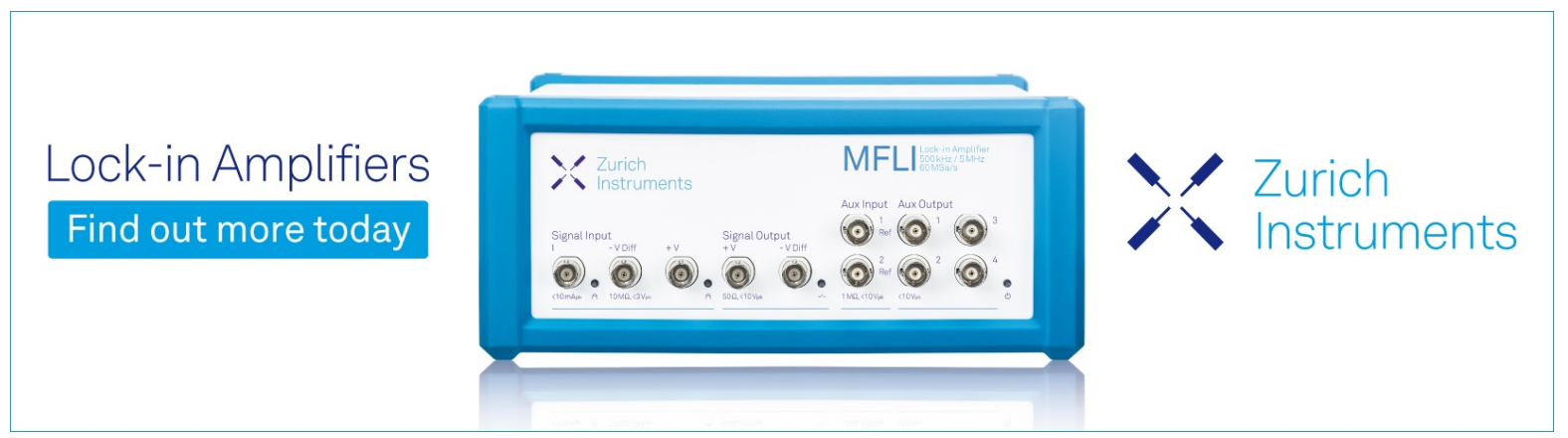

Zurich

- Instruments 


\title{
Experimental and theoretical analysis for total electron scattering cross sections of benzene
}

\author{
Cite as: J. Chem. Phys. 151, 084310 (2019); doi: 10.1063/1.5116076 \\ Submitted: 21 June 2019 - Accepted: 7 August 2019 • \\ Published Online: 30 August 2019
}

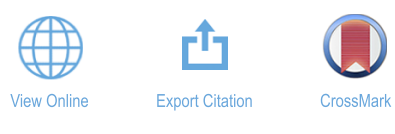

\author{
F. Costa, ${ }^{1}$ L. Álvarez, ${ }^{1}$ A. I. Lozano, ${ }^{1,2}$ F. Blanco, ${ }^{3}$ (D) J. C. Oller, \\ M. H. F. Bettega, ${ }^{5}$ (iD Ferreira da Silva, ${ }^{2}$ (D) P. Limão-Vieira, \\ and G. García \\ A. Muñoz, ${ }^{4}$ A. Souza Barbosa, \\ R. D. White, ${ }^{6}$ M. J. Brunger, ${ }^{7}$
}

\begin{abstract}
AFFILIATIONS
${ }^{1}$ Instituto de Física Fundamental, Consejo Superior de Investigaciones Científicas, Serrano 113-bis, 28006 Madrid, Spain

${ }^{2}$ Laboratório de Colisões Atómicas e Moleculares, CEFITEC, Departamento de Física, Universidade NOVA de Lisboa, 2829-516 Caparica, Portugal

${ }_{3}^{3}$ Departamento de Estructura de la Materia Física Térmica y Electrónica, Universidad Complutense de Madrid, Plaza de Ciencias 1, 28040 Madrid, Spain

«Departamento de Tecnología, Centro de Investigaciones Energéticas, Medioambientales y Tecnológicas (CIEMAT), Avenida Complutense 22, 28040 Madrid, Spain

${ }^{5}$ Departamento de Física, Universidade Federal do Paraná, CP 19044, 81531-990 Curitiba, Paraná, Brazil

${ }^{6}$ College of Science and Engineering, James Cook University, Townsville, Australia

${ }^{7}$ College of Science and Engineering, Flinders University, GPO Box 2100, Adelaide, SA 5001, Australia

${ }^{8}$ Centre for Medical Radiation Physics, University of Wollongong, NSW 2522, Australia
\end{abstract}

a)g.garcia@csic.es

\begin{abstract}
Measurements of the total electron scattering cross sections (TCSs) from benzene, in the impact energy range of 1-1000 eV, are presented here by combining two different experimental systems. The first utilizes a magnetically confined electron transmission beam for the lower energies (1-300 eV), while the second utilizes a linear transmission beam apparatus for the higher energies (100-1000 eV). These cross sections have also been calculated by means of two different theoretical methods, the Schwinger Multichannel with Pseudo Potential (SMCPP) procedure, employing two different approaches to account for the polarization of the target for impact energies between 0.1 and $15 \mathrm{eV}$, and the Independent Atom Model with the Screening Corrected Additivity Rule including Interference effect (IAM-SCAR+I) paradigm to cover the 10-10000 eV impact energy range. The present results are compared with available theoretical and experimental data, with the level of accord being good in some cases and less satisfactory in others, and some predicted resonances have been identified. In particular, we found a $\pi^{*}$ shape resonance at $1.4 \mathrm{eV}$ and another feature in the energy region $4.6-4.9 \mathrm{eV}$ interpreted as a $\pi^{*}$ resonance $\left({ }^{2} \mathrm{~B}_{2 \mathrm{~g}}\right.$ symmetry), which is a mixture of shape and a core excited resonance, as well as a Feshbach resonance at $5.87 \mathrm{eV}$ associated with the $3 \mathrm{~s}\left(\mathrm{a}_{1 \mathrm{~g}}\right.$ ) Rydberg state. A Born-type formula to extrapolate TCS values for energies above $10000 \mathrm{eV}$ is also given. This study provides a complete set of TCS data, with uncertainty limits within $10 \%$, ready to be used for modeling electron transport applications.
\end{abstract}

Published under license by AIP Publishing. https://doi.org/10.1063/1.5116076

\section{INTRODUCTION}

In recent years, a great effort has been made to characterize radiation damage at the molecular level. ${ }^{1}$ Since radiobiological effects of radiation are triggered by initial molecular alterations, mostly produced by atomic and molecular radical species ${ }^{2}$ and secondary electron interactions, ${ }^{3}$ electron scattering processes from biologically relevant molecules (DNA and RNA bases, and molecular analogs) have recently been studied both from the theoretical and experimental points of view. Within these DNA and RNA analogs, we could mention the molecules pyrimidine, pyridazine, pyridine, and benzoquinone in which we have recently been 
interested. ${ }^{4-7}$ Other relevant biomolecules are those used as treatment complements, as in the case of drug precursors and anesthetics (thiophene ${ }^{8,9}$ and sevoflurane ${ }^{10}$ ) or radiosensitizers (nitroimidazole $^{11}$ and methyl nitroimidazole ${ }^{12}$ ). What the biomedical community ultimately needs is a modeling procedure, based on realistic interaction probabilities (i.e., cross sections), which is able to describe the effect of the incident radiation not only in terms of the energy deposition but also in accounting for dissociative processes which may lead to biological damage. Such modeling procedures are essentially event-by-event Monte Carlo simulation programs, which, at least in part, use as input parameters the cross sections associated with all the possible scattering processes that may occur in the considered energy range. These input parameters constitute a comprehensive and consistent data set, which requires benchmarking against molecular prototypes. ${ }^{13}$ One of the simplest molecular structures, from which many of the aforementioned biologically relevant molecules might be considered as chemical derivatives, is the benzene ring. In this context, the main goal of the present collaborative study is to determine a complete cross section data base for benzene in order to obtain a benchmark reference for modeling purposes. In addition, as many of the previously mentioned biomolecules have a permanent dipole moment, which strongly affects the scattering dynamics, we will later extend this initial benzene study to nitrobenzene, a benzene derivative possessing a large permanent dipole moment due to its $\mathrm{NO}_{2}$ functional group. This paper constitutes the first part of the aforementioned long-term collaborative study and is focused on the total electron scattering cross sections (TCSs) from benzene $\left(\mathrm{C}_{6} \mathrm{H}_{6}\right)$. TCSs are important values to validate cross section data sets. This follows as they represent the sum of the cross sections for all the energetically accessible processes (open channels) ${ }^{14}$ at a given energy, and therefore, they constitute a reference value to estimate the completeness and accuracy of the corresponding partial cross sections.

Benzene is a nonpolar molecule with an extremely large dipole polarizability ( $\alpha$ ). It participates in several synthetic processes employed by both the pharmaceutical and petrochemical industries. Indeed, in many respects it can be considered as the prototype aromatic system, displaying conjugative, delocalization, and resonance effects, thereby making it an ideal species with which to study such phenomena.

The original measurements of TCSs at low energies $(0.5-25 \mathrm{eV})$ for electron-benzene collisions, using a circular Ramsauer apparatus, were published in 1931 by Holst and Holtsmark. ${ }^{16}$ In 1988, Sueoka ${ }^{17}$ employed a linear-transmission-beam technique to obtain TCS values from 1 to $400 \mathrm{eV}$ electron impact energies. Subsequently, Możejko et al. ${ }^{18}$ applied a similar technique but utilized an electron monochromator to improve the energy resolution at lower energies $(0.6-250 \mathrm{eV})$ and a modified Ramsauer apparatus to extend the energy range from 90 to $3500 \mathrm{eV}$. They thus obtained TCS values from 0.6 to $3500 \mathrm{eV}$, the components of which being found to be in good agreement within the overlapping energies. With even better energy resolution, as provided by a photoelectron beam generated by synchrotron radiation, Gulley et al. ${ }^{19}$ extended the available TCS measurements down to $0.035 \mathrm{eV}$. A critical discussion on the bona fides of these early experiments can be found in the review of Karwasz et al. ${ }^{20}$ More recently, both Makochekanwa et al. ${ }^{21}$ and Kimura et al. ${ }^{22}$ revisited Sueoka's measurements using an improved experimental arrangement.
From the theoretical point of view, Gianturco and Lucchese $^{23}$ used a model potential method to calculate integral elastic cross sections, and one-electron resonances in benzene for impact electron energies from 0.001 to $30 \mathrm{eV}$. Note that within a fixed nuclei representation, and below the electronic excitation threshold (around $4 \mathrm{eV}$ ), these data are equivalent to the total electron scattering cross sections, as vibrational excitation is effectively ignored. Bettega et al. ${ }^{24}$ employed the Schwinger multichannel (SMC) method to compute elastic integral and differential cross sections for the scattering of electrons by benzene in the static-exchange and static-exchange plus polarization approximations and discussed its resonance spectra. Within the independent atom model (IAM) and using different versions of the additivity rule (AR), Jiang et al., ${ }^{25}$ for impact energies between 30 and $3000 \mathrm{eV}$, and Sun et al., ${ }^{26}$ in the 10-2000 eV energy range, calculated the electron-benzene TCS. Singh et al. ${ }^{27}$ also included results of TCS calculations for electrons scattering off benzene from 10 to $5000 \mathrm{eV}$ impact energies in their benzene derivatives analysis by using a modified spherical complex optical potential (MSCOP) method. Recently, Barbosa and Bettega ${ }^{28}$ employed the Schwinger multichannel method implemented with pseudopotentials (SMCPP) to carry out systematic elastic cross section calculations including a complete resonance analysis to identify any temporary anion formation and the presence of a virtual state and a Ramsauer-Townsend minimum. Finally, Prajapati et al. ${ }^{29}$ computed the electron scattering TCS for benzene by using two different methods. The first was a R-matrix procedure provided by the Quantemol-N software $^{29}$ package for the lower energies $(0.01-20 \mathrm{eV})$, while the second applied the SCOP formalism from the ionization threshold up to $5000 \mathrm{eV}$.

The experimental contribution of the present study consists of two sets of TCS data as measured with two different apparatus. For the lower energies (1-300 eV), we used a magnetically confined electron transmission beam system with an energy resolution of about $200 \mathrm{meV}$, while for the higher energies $(100-1000 \mathrm{eV})$, a modified transmission-beam system with improved angular resolution $\left(<10^{-5} \mathrm{sr}\right)$ has been utilized. Concerning our theoretical data, we have re-examined our previous SMCPP calculation ${ }^{28}$ in the energy range where they can be considered to be roughly equivalent to the TCS $(0-5 \mathrm{eV})$. Note that in this range, below the electronic excitation threshold, the rotational excitation cross sections are much lower in magnitude than the elastic cross sections, and the electron attachment cross sections are accounted by the SMCPP calculation as resonances over the integral elastic cross sections. We also present total electron scattering cross sections in the $(1-10000 \mathrm{eV})$ energy range as calculated with our independent atom model complemented with the screening corrected additivity rule ${ }^{30,31}$ and including interference effects (IAM-SCAR+I). ${ }^{32}$ From a detailed comparison between the measured TCS local maxima and the calculated SMCPP integral elastic cross sections, the position and characteristics of several low-lying resonances are analyzed.

The remaining sections of this article are organized as follows: In Sec. II, we describe our experimental techniques, including an analysis of possible uncertainty sources. The theoretical methods used to calculate the low energy integral elastic cross sections and total electron scattering cross sections, over the whole energy range considered here, are very briefly summarized in 
Sec. III. The obtained experimental and theoretical TCS values are presented in Sec. IV, which also includes a discussion of the present results in comparison with those from previous studies. Finally, some conclusions are drawn in Sec. V.

\section{EXPERIMENTAL ARRANGEMENTS}

As noted earlier, two different experimental systems have been used in order to accurately cover the incident electron energy range considered in this study.

\section{A. Magnetically confined electron beam (MCEB) system}

The experimental apparatus employed to measure the TCS from low to intermediate energies $(1-300 \mathrm{eV})$ has already been detailed in a previous publication. ${ }^{33}$ Basically, it consists of a pulsed linear transmission beam system with a strong axial magnetic field $(0.1 \mathrm{~T})$ confinement. Under these conditions, we can assume that every scattering event is converted into a kinetic energy loss in the forward direction that can be measured with a retarding field analyzer (see Ref. 33 for full details). Note that any electrons scattered at angles higher than $90^{\circ}$ move back to the cathode, where they are again reflected toward the interaction region. As explained in Ref. 33, backscattered electrons affect the energy resolution and their contribution to the experimental uncertainty limit will be discussed below.

The electron beam is produced by an emitting thermionic tungsten filament and focused along the magnetic field axis onto the entrance aperture of a gas cell (GC) containing molecular nitrogen. This chamber is a cooling electron trap, where the energy spread of the beam $(\Delta E)$ is reduced down to $200 \mathrm{meV}$ through successive collisions with $\mathrm{N}_{2}$ molecules. The primary electron beam is then pulsed and accelerated/decelerated to the required incident energy $(E)$ at the entrance aperture ( $1.5 \mathrm{~mm}$ in diameter) of the scattering chamber (SC), which contains the target molecules (benzene in this case) at a well-known pressure. A $1.5 \mathrm{~mm}$ exit aperture defines the collision length $(L=40 \mathrm{~mm})$ along the SC. Benzene is introduced into the SC through a leak valve, where it is maintained at a constant pressure as measured with a MKS-Baratron $627 \mathrm{~B}$ absolute capacitance manometer. The gas pressure was varied from 0 to 3 mTorr during the measurements, and the transmitted intensity was recorded for at least 5 different pressure values within this range. Electrons emerging from the SC are energy-selected by a retarding potential energy analyzer (RPA) and finally detected by a double microchannel plate (MCP) electron multiplier operating in single counting mode.

The experimental electron total scattering cross section $\left(\sigma_{t}\right)$ is obtained for each incident electron energy from the Beer-Lambert attenuation law,

$$
I=I_{0} e^{-n \sigma_{t} L},
$$

where $I$ is the transmitted electron intensity, $I_{0}$ is the initial intensity when there is no gas in the SC, $n$ is the benzene gas density, and $L$ is the length of the collision chamber. Assuming an ideal gas behavior, Eq. (1) can be written as

$$
\ln \left(\frac{I}{I_{0}}\right)=-L \sigma_{t} n=-\frac{L p}{k T} \sigma_{t}
$$

In Eq. (2), $k$ is Boltzmann's constant, $T$ is the absolute temperature, and $p$ is the benzene gas pressure. $T$ is derived from $T=\sqrt{T_{c} T_{m}}$, where $T_{c}$ is the temperature of the collision chamber (measured with a calibrated thermocouple) and $T_{m}$ is the temperature of the Baratron manometer. In the conditions of this experiment, $T_{\mathrm{c}} \approx T_{\mathrm{m}}$ and therefore thermal transpiration effects are practically negligible. The accuracy on the pressure measurements is assumed to be better than $1 \%$, as stated by the manufacturer. The entire measurement conditions, data acquisition, and data analysis are monitored and controlled by a custom designed LabView (National Instruments) program.

The total experimental (random) uncertainty limits on these measurements are within $5 \%$, as derived by adding in quadrature all the known uncertainty sources: a statistical uncertainty (our measurements were repeated at least 5 times to ensure standard deviations below $4.5 \%)$, the uncertainty in the pressure measurement (1\%), energy calibrations (1\%), and the scattering length determination $(1.5 \%)$. In the conditions of this experiment, the magnetic beam intensity along the scattering chamber ensures a cyclotron radius of the colliding electron that is less than $0.5 \mathrm{~mm}$ for the whole energy range considered here $(1-300 \mathrm{eV})$. This means that the effective diameter of the electron beam in the SC is lower than the entrance and exit aperture diameters, so ensuring that no collimating effects are distorting the present measurements. For the low pressure conditions of those measurements, Eq. (2) was fitted to a single exponential function, with 0.999 correlation index thus indicating that multiple scattering effects are not affecting the present results. Transmitted intensities, for benzene pressures ranging from 0 to 3 mTorr, typically varied from $2 \times 10^{3}$ to $0.7 \times 10^{2}$ electrons/s, which corresponds to equivalent electron currents from $3 \times 10^{-16}$ to $1.1 \times 10^{-17} \mathrm{~A}$. For such low current conditions, no dependence of the cross sections on the electron current was observed, which indicates that space charge effects are not present in our measurements.

As discussed in detail in Ref. 33, there is a systematic error inherent to all magnetically confined experimental systems. As a consequence of the gyromagnetic motion of the scattered electrons, imposed by the axial magnetic field, the angular resolution (acceptance angle of the detector, $\Delta \theta^{\circ}$ ) is related to the energy resolution (energy spread, $\Delta E$ ) and the incident energy $(E)$ according to the expression,

$$
\Delta \theta^{\circ}=\arccos \sqrt{1-\frac{\Delta E}{E}} .
$$

In these conditions, electrons elastically scattered into the $\Delta \theta^{\circ}$ and $180-\Delta \theta^{\circ}$ angles (the so-called experimental "missing" angles) are considered by the detector as unscattered electrons and therefore tend to lower the measured cross sections from their true value. This effect always acts in the same direction, i.e., tending to lower the total cross section, and its magnitude can be evaluated by integrating calculated differential elastic cross section $\left(D C S_{e l}\right)$ values over these acceptance angles. Although it is not the case for nonpolar benzene, for polar molecules the same argument applies to the differential rotational excitation cross sections ${ }^{33}\left(D C S_{\text {rot }}\right)$. The total decrease in the scattering cross section $[\sigma(\Delta \theta)]$, due to the experimental "missing angles," can be estimated with the following expression: $:^{34,35}$ 


$$
\begin{aligned}
\sigma(\Delta \theta)= & 2 \pi\left[\int_{0}^{\Delta \theta}\left(D C S_{e l}+D C S_{r o t}\right) \sin \theta d \theta\right. \\
& \left.+\int_{180-\Delta \theta}^{180}\left(D C S_{e l}+D C S_{r o t}\right) \sin \theta d \theta\right] .
\end{aligned}
$$

Using our calculated DCS and the $\Delta \theta$ values given by Eq. (3), the contribution of this systematic error increases from $3 \%$ to $10 \%$ when the incident energy decreases from 300 to $1 \mathrm{eV}$. These observations should be taken into consideration when further comparisons between experimental and theoretical values are made in Sec. IV.

\section{B. Linear transmission-beam (LTB) system}

A schematic diagram of the experimental configuration used for intermediate and high energies is shown in Fig. 1. It is based on that used in previous studies ${ }^{6,36}$ but presents some modifications in order to adapt the system to the requirements of the present measurements. The electron beam is generated by a negatively biased $\left(V_{\mathrm{C}}\right)$ thoriated tungsten hairpin filament, being focused and then deflected toward the scattering chamber (SC). Typical electron currents were $10^{-7} \mathrm{~A}$ with an energy spread of about $600 \mathrm{meV}$. The SC consists of a $50 \times 50 \times 50 \mathrm{~mm}^{3}$ metallic (Dural) cube, defined by two $2 \mathrm{~mm}$ diameter apertures which are separated by a $50 \mathrm{~mm}$ length (L). At the entrance of the SC, two further $1.5 \mathrm{~mm}$ collimators ensure that the electron beam diameter at this entrance is less than the collimator diameter so preventing possible gas focusing effects. The SC is perpendicularly held to the gas inlet flange through a $32 \mathrm{~mm}$ diameter polytetrafluoroethylene (PTFE) tube connected to a MKS-Baratron (627B) absolute capacitance manometer, so maintaining a steady gas flow during the measurements. With this configuration, the SC is not grounded; rather, it is connected to a biasing $\pm 1000 \mathrm{~V}$ power supply $\left(V_{\mathrm{SC}}\right)$. The kinetic energy $(E)$ inside the SC is then defined by $E=V_{\mathrm{C}}-V_{\mathrm{SC}}$. Electrons emerging from the SC pass through a deflecting plate and a lens tube system and then are analyzed in energy with a hemispherical electrostatic spectrometer. Transmitted electrons are finally detected with a two-stage microchannel plate (MCP) operating in single counting mode.

The regions that include both the electron gun and the scattering chamber, and the region of the analyzer, are differentially pumped by two turbo-pumps and reach a background pressure of about $10^{-8}$ Torr. This allowed us to maintain an overall pressure in the system below $10^{-7}$ Torr during the measurements, while the pressure in the scattering chamber was varied between 1 and 5 mTorr.

As with the previous MCEB system, the transmitted beam intensity follows the Beer-Lambert law [Eq. (1)]. According to Eq. (2), the total cross sections can be derived from the slope of a semilogarithmic plot of the observed attenuation $\left(I / I_{0}\right)$ as a function of the gas pressure $(P)$. As also for the MCEB experiment, the temperature $(T)$ is derived from $T=\sqrt{T_{C} T_{m}}$, where $T_{\mathrm{C}}$ and $T_{\mathrm{m}}$ are the temperature of the scattering chamber, as measured with a thermocouple, and the Baratron gauge operating temperature. The accuracy of our pressure measurements is again assumed to be better than $1 \%$, as stated by the MKS Baratron manufacturer.

One relevant feature of this apparatus is the angular acceptance of the hemispherical spectrometer, which is used as the energy analyzer of the transmitted electrons. The $1.5 \mathrm{~mm}$ diameter entrance aperture of the analyzer is placed at $400 \mathrm{~mm}$ from the center of the SC so that the solid angle subtended by the detector is of the order $10^{-5} \mathrm{sr}$ leading to a practical acceptance angle of about $0.25^{\circ}$ (the lens tube is grounded during the TCS measurements).

The analyzer is remotely controlled by a PC running a suitable custom LabView (National Instruments) program, in order to record the EEL spectra and the attenuation of the primary beam as a function of the gas pressure in the SC. Using these data, the LabView program provides the attenuation plots, the analysis fitting procedure, and the corresponding TCS values resulting from the fitted attenuation plots.

Each cross section measurement was repeated at least five times to ensure statistical uncertainties below $4 \%$. Further note that the overall energy resolution here was determined by the energy spread of the incident electron beam, i.e., $600 \mathrm{meV}$.

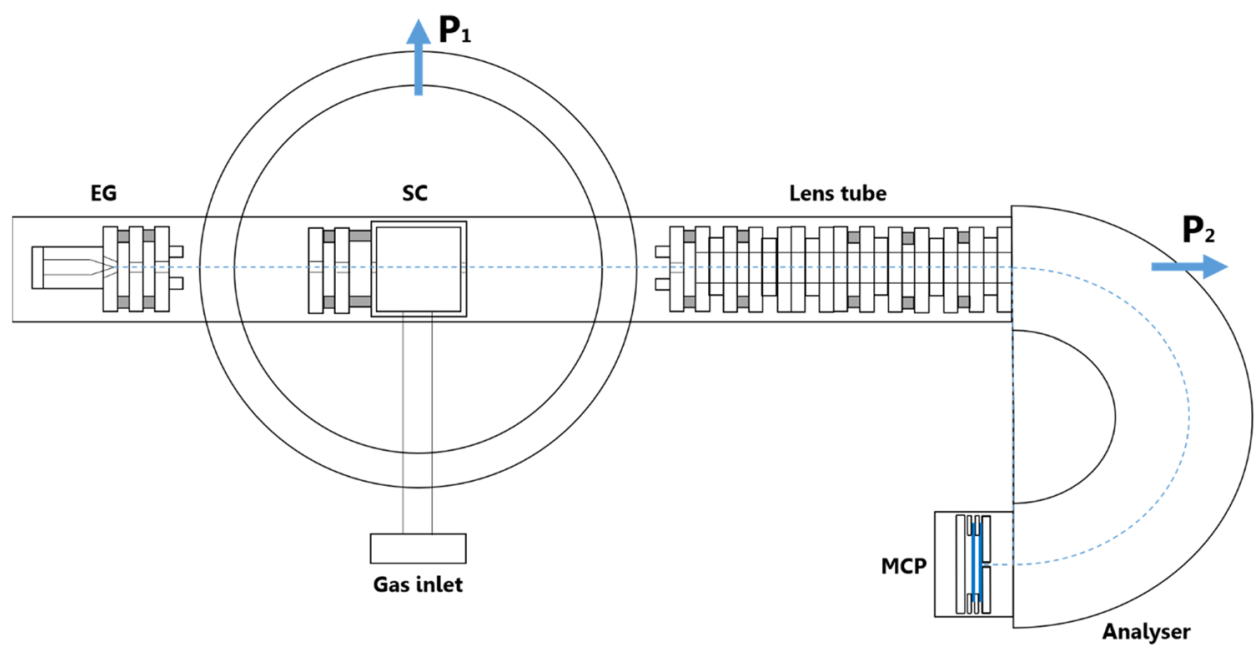

FIG. 1. Diagram and current configuration of the linear transmission-beam system. EG corresponds to the electron gun, SC corresponds to the scattering chamber, Gas inlet refers to the valve system, the lens tube is composed of several lenses that guide the beam toward the hemispherical analyzer, P1 and P2 correspond to two different turbo pumps, and the MCP (microchannel plate) is where the transmitted electrons are ultimately detected. 
As already mentioned, a significant possible source of systematic error in transmission-beam measurements results from electrons scattered in the forward direction that are not discriminated against by the energy analyzer. These are due in principle to those elastic, rotational excitation, and ground state vibrational excitation processes (i.e., those open channels given our energy resolution) for which the electron is scattered into the acceptance angle of the energy analyzer. The contribution of this effect to the present LTB measurements was determined to be less than $1 \%$, by integrating the calculated elastic DCS over the acceptance angle for each incident energy (note that rotational excitations are not determined for a nonpolar molecule such as benzene and the vibrational excitations of the ground state can be neglected for the relatively high impact energies considered in this case). Due to the geometry of the present experiment, other sources of systematic error are connected with the assumption that the actual absorption length (l) is coincident with the geometrical length of the scattering chamber (L), and with possible pressure gradients between the electron interaction region and the Baratron gauge position which may originate from the differential pumping system. In order to check if any of these systematic error sources is affecting our experimental results, we measured from time to time ( 21 times in total during the whole experiment) the well-known electron scattering TCS for $\mathrm{N}_{2}$ at $100 \mathrm{eV}$ collision energy. We obtained an average value of $(8.89 \pm 0.40) \times 10^{-20} \mathrm{~m}^{2}$, which is in excellent agreement with the reference value given by Itikawa $^{37}$ of $8.94 \times 10^{-20} \mathrm{~m}^{2}$.

Combining all the above uncertainty sources, the total uncertainty limits of the present experimental TCSs for the incident energy range considered with the LTB system $(100-1000 \mathrm{eV})$ are estimated to be within $\pm 5 \%$.

\section{THEORETICAL METHODS}

As already noted, in order to cover the incident electron energy range considered in this study, we have employed two different theoretical methods, the $\mathrm{SMCPP}^{38}$ to calculate the elastic integral cross sections (ICSs) from 0 to $15 \mathrm{eV}$ and the IAM-SCAR $+\mathrm{I}^{32}$ to obtain the total electron scattering cross sections from 1 to $10000 \mathrm{eV}$. Both methods have been extensively described in previous articles (see Refs. 28 and 32 and references therein), and therefore, we will not repeat here the details of these calculations. Briefly, the SMCPP is a variational method to solve the scattering equations which considers the static and exchange interactions. In this application, this allows for two different levels of approach to include polarization effects (SEP1 and SEP2). ${ }^{28}$ Within this representation, shape resonances can be identified from the integral elastic cross section profile, which we reiterate in this case has been calculated for very low impact energies from 0 to $15 \mathrm{eV}$. The IAM-SCAR+I method is a well-established calculation procedure which is based on an independent atom representation but considers the molecular geometry to account for the overlapping of the atomic cross sections and additionally considers multiple scattering interference effects. ${ }^{30-32}$

\section{RESULTS AND DISCUSSION}

The present experimental TCS values for benzene, in the incident energy range of 1-300 eV and as measured with the MCEB system, are shown in Table I together with their uncertainty and energy and angular resolution limits. Our experimental results and their uncertainty limits for impact energies between 100 and $1000 \mathrm{eV}$, as obtained with the LTB apparatus, are shown in Table II. Note that both sets of experimental results agree, to within the uncertainty limits, in the overlapping energy range (100-300 eV). On the other hand, our present theoretical data, as obtained with both calculation methods described above, are shown in Table III and plotted in Fig. 2. Below $10 \mathrm{eV}$, our IAM-SCAR+I approach is not accurate, and only qualitative information can be expected from it for such low energies (see Fig. 2). As mentioned above, our SMCPP calculation gives the integral elastic cross sections, but below $7 \mathrm{eV}$, where electronic excitations are not significant, we can consider that they are approximately equivalent to the TCS (i.e., we assume vibrational excitation is small ${ }^{39}$ ). As shown in Fig. 2, for incident energies within $7-10 \mathrm{eV}$, there is a fair concordance between the results given by our two calculation methods. This allows, with only a minor upward scaling of the SMCPP (SEP2) result, our combining them to derive a complete set of calculated TCSs over the whole energy range $(0.1-$ $10000 \mathrm{eV}$ ). As the merged data (labeled in Fig. 3 as SMCPP-SCAR) are based on both calculations in their respective energy ranges of validity, we can estimate for them an uncertainty limit of $\pm 10 \%$. This figure is based on results from previous comparisons between calculated and experimental data for similar targets including pyridine $^{5,6}$ and $p$-benzoquinone. Comparing with the other available calculations (see Fig. 2), apart from the resonances that will be discussed later, Gianturco and Lucchese ${ }^{23}$ give integral elastic cross sections which are lower in magnitude than ours, for the energies where they are roughly equivalent to the TCSs, with a maximum discrepancy of about $20 \%$ at around $5 \mathrm{eV}$. As expected, the additivity rule (AR) method used by Jiang et al., ${ }^{25}$ even with some energy dependent correction, fails below $100 \mathrm{eV}$ where they overestimate the total cross section values. Since this method does not consider interference terms, ${ }^{32}$ it tends to give TCS values lower than our SMC-SCAR hybrid for higher energies $(>100 \mathrm{eV})$, being about $50 \%$ lower in magnitude than ours at $3000 \mathrm{eV}$. Sun et al. ${ }^{26}$ proposed a modified additivity rule method, aiming to improve its reliability for the lower energies. Sun's results ${ }^{26}$ are almost coincident with those from Jiang et al..$^{25}$ for energies above $100 \mathrm{eV}$, but they correct Jiang's data $^{25}$ below $100 \mathrm{eV}$ now giving TCS values in good agreement with those of the present calculation down to $10 \mathrm{eV}$. The so-called MSCOP method of Singh et al. ${ }^{27}$ provided TCS values for impact energies between 10 and $5000 \mathrm{eV}$. It is basically a single center optical potential method, applied to different groups within the molecule, to finally generate a cross section by adding the corresponding results for each group. ${ }^{27}$ TCSs calculated by Singh et al. ${ }^{27}$ are higher than ours by about $50 \%-60 \%$ between 10 and $100 \mathrm{eV}$ but tend to converge to ours for increasing energies. Finally, Prajapati et al. ${ }^{29}$ used a similar SCOP method in combination with an R-matrix calculation ${ }^{40}$ based on the commercial Quantemol- $\mathrm{N}^{41}$ software package. Their results for the lower energies (corresponding to the Quantemol$\mathrm{N}$ code) are in clear disagreement with those from our SMCPP method (see Fig. 2). However, their SCOP results agree reasonably well with our IAM-SCAR+I values from 20 to $1000 \mathrm{eV}$, although their energy dependence ${ }^{29}$ for increasing energies tends to diverge from ours.

In order to compare our results with those from previous measurements, the present experimental and theoretical TCS results, in particular our hybrid theoretical TCS result, are plotted in Fig. 3 
TABLE I. Experimental total electron scattering cross section data in benzene for low to medium energies, from the MCEB system, with their uncertainty, energy $(\Delta E)$, and angular $(\Delta \Theta)$ resolution limits.

\begin{tabular}{|c|c|c|c|c|c|}
\hline \multicolumn{6}{|c|}{ Magnetically confined system } \\
\hline Energy $(\mathrm{eV})$ & $\sigma_{t}\left(10^{-20} \mathrm{~m}^{2}\right)$ & $\begin{array}{c}\text { Statistical } \\
\text { uncertainty }(\%)\end{array}$ & $\begin{array}{c}\text { Absolute total } \\
\text { uncertainty }( \pm)\end{array}$ & $\Delta E(\mathrm{eV})$ & $\Delta \theta(\mathrm{deg})$ \\
\hline 1.0 & 30.2 & 2.6 & 0.8 & 0.2 & 26.6 \\
\hline 1.2 & 32.2 & 1.6 & 0.5 & 0.17 & 22.1 \\
\hline 1.4 & 35.0 & 3.4 & 1.4 & 0.2 & 22.2 \\
\hline 1.6 & 32.5 & 3.7 & 1.2 & 0.2 & 20.7 \\
\hline 1.8 & 32.9 & 1.5 & 0.5 & 0.2 & 19.5 \\
\hline 2.0 & 32.4 & 2.2 & 0.7 & 0.2 & 18.4 \\
\hline 2.2 & 31.5 & 3.8 & 1.2 & 0.2 & 17.5 \\
\hline 2.4 & 30.2 & 4.6 & 1.4 & 0.2 & 16.8 \\
\hline 2.6 & 31.6 & 3.8 & 1.2 & 0.2 & 16.1 \\
\hline 2.8 & 33.8 & 3.0 & 1.0 & 0.2 & 15.5 \\
\hline 3.0 & 35.7 & 3.6 & 1.3 & 0.2 & 15.0 \\
\hline 3.2 & 36.9 & 2.7 & 1.0 & 0.2 & 14.5 \\
\hline 3.4 & 38.0 & 4.2 & 1.6 & 0.21 & 14.4 \\
\hline 3.6 & 38.4 & 2.1 & 0.8 & 0.2 & 13.6 \\
\hline 3.8 & 38.7 & 2.8 & 1.1 & 0.21 & 13.6 \\
\hline 4.0 & 39.7 & 3.5 & 1.4 & 0.2 & 12.9 \\
\hline 4.2 & 41.9 & 3.3 & 1.4 & 0.2 & 12.6 \\
\hline 4.4 & 43.4 & 1.3 & 0.6 & 0.2 & 12.3 \\
\hline 4.6 & 46.4 & 2.8 & 1.3 & 0.2 & 12.0 \\
\hline 4.8 & 43.8 & 2.3 & 1.0 & 0.2 & 11.8 \\
\hline 5.0 & 45.5 & 2.6 & 1.2 & 0.2 & 11.5 \\
\hline 5.2 & 44.4 & 2.3 & 1.0 & 0.22 & 11.9 \\
\hline 5.4 & 45.3 & 3.5 & 1.6 & 0.22 & 11.6 \\
\hline 5.7 & 48.2 & 3.1 & 1.5 & 0.2 & 10.8 \\
\hline 6.0 & 45.4 & 3.7 & 1.7 & 0.22 & 11.0 \\
\hline 6.2 & 46.3 & 1.3 & 0.6 & 0.2 & 10.3 \\
\hline 6.5 & 46.5 & 4.3 & 2.0 & 0.2 & 10.1 \\
\hline 7.0 & 46.4 & 3.9 & 1.8 & 0.2 & 9.7 \\
\hline 7.3 & 48.8 & 2.7 & 1.3 & 0.21 & 9.8 \\
\hline 7.5 & 50.4 & 4.4 & 2.2 & 0.2 & 9.4 \\
\hline 7.7 & 52.3 & 3.1 & 1.6 & 0.2 & 9.3 \\
\hline 8.0 & 52.9 & 1.9 & 1.0 & 0.21 & 9.3 \\
\hline 8.5 & 54.3 & 1.7 & 0.9 & 0.2 & 8.8 \\
\hline 8.7 & 56.6 & 2.8 & 1.6 & 0.2 & 8.7 \\
\hline 9.0 & 59.7 & 3.9 & 2.3 & 0.2 & 8.6 \\
\hline 9.5 & 60.8 & 2.3 & 1.4 & 0.2 & 8.3 \\
\hline 10 & 58.7 & 2.7 & 1.6 & 0.2 & 8.1 \\
\hline 11 & 58.8 & 1.2 & 0.7 & 0.2 & 7.7 \\
\hline 11.5 & 57.6 & 1.6 & 0.9 & 0.2 & 7.6 \\
\hline 12 & 55.8 & 0.9 & 0.5 & 0.2 & 7.4 \\
\hline 13 & 54.9 & 2.0 & 1.1 & 0.2 & 7.1 \\
\hline 14 & 53.7 & 3.4 & 1.8 & 0.2 & 6.9 \\
\hline 15 & 53.2 & 2.3 & 1.2 & 0.19 & 6.5 \\
\hline 16 & 52.7 & 1.5 & 0.8 & 0.2 & 6.4 \\
\hline 16.5 & 51.3 & 1.2 & 0.6 & 0.2 & 6.3 \\
\hline 17 & 49.5 & 3.4 & 1.7 & 0.2 & 6.2 \\
\hline 17.5 & 51.2 & 1.2 & 0.6 & 0.2 & 6.1 \\
\hline 18 & 52.9 & 3.2 & 1.7 & 0.19 & 5.9 \\
\hline 18.5 & 51.8 & 1.9 & 1.0 & 0.2 & 6.0 \\
\hline
\end{tabular}


TABLE I. (Continued.)

\begin{tabular}{|c|c|c|c|c|c|}
\hline \multicolumn{6}{|c|}{ Magnetically confined system } \\
\hline Energy (eV) & $\sigma_{t}\left(10^{-20} \mathrm{~m}^{2}\right)$ & $\begin{array}{c}\text { Statistical } \\
\text { uncertainty }(\%)\end{array}$ & $\begin{array}{c}\text { Absolute total } \\
\text { uncertainty }( \pm)\end{array}$ & $\Delta E(\mathrm{eV})$ & $\Delta \theta(\operatorname{deg})$ \\
\hline 19 & 51.0 & 3.5 & 1.8 & 0.19 & 5.7 \\
\hline 20 & 49.7 & 4.0 & 2.0 & 0.18 & 5.4 \\
\hline 22 & 48.4 & 2.0 & 1.0 & 0.19 & 5.3 \\
\hline 25 & 47.9 & 1.0 & 0.5 & 0.19 & 5.0 \\
\hline 30 & 46.6 & 0.6 & 0.3 & 0.2 & 4.7 \\
\hline 35 & 46.1 & 1.1 & 0.5 & 0.22 & 4.5 \\
\hline 40 & 44.1 & 2.7 & 1.2 & 0.22 & 4.3 \\
\hline 45 & 43.0 & 3.3 & 1.4 & 0.22 & 4.0 \\
\hline 50 & 40.7 & 2.2 & 0.9 & 0.25 & 4.1 \\
\hline 60 & 38.8 & 0.5 & 0.2 & 0.26 & 3.8 \\
\hline 70 & 36.8 & 3.5 & 1.3 & 0.26 & 3.5 \\
\hline 80 & 34.9 & 2.0 & 0.7 & 0.25 & 3.2 \\
\hline 90 & 34.0 & 2.9 & 1.0 & 0.25 & 3.0 \\
\hline 100 & 32.6 & 1.5 & 0.5 & 0.3 & 3.1 \\
\hline 120 & 30.9 & 1.0 & 0.3 & 0.3 & 2.9 \\
\hline 150 & 28.6 & 0.7 & 0.2 & 0.3 & 2.6 \\
\hline 200 & 26.6 & 3.4 & 0.9 & 0.3 & 2.2 \\
\hline 250 & 23.9 & 2.1 & 0.5 & 0.3 & 2.0 \\
\hline 300 & 21.4 & 3.3 & 0.7 & 0.3 & 1.8 \\
\hline
\end{tabular}

together with those experimental values available in the literature for incident energies ranging from 1 to $1000 \mathrm{eV}$. Early measurements from Sueoka ${ }^{17}$ show a reasonable agreement (to within $\sim 10 \%$ ) for the lower energies, from 1 to $7 \mathrm{eV}$. Above this energy, however, his TCS values are systematically lower than the present data by about $25 \%$. This discrepancy may be attributed, at least in part, to the poorer energy resolution of the TOF analyzer used in Ref. 17.

TABLE II. Experimental total electron scattering cross section data in benzene for medium to high energies, from the LTB system, with their error estimates.

\begin{tabular}{lcc}
\hline \hline \multicolumn{3}{c}{ Linear transmission-beam system } \\
\hline Energy $(\mathrm{eV})$ & $\sigma_{t}\left(10^{-20} \mathrm{~m}^{2}\right)$ & Absolute total uncertainty $( \pm)$ \\
\hline 100 & 36.53 & 1.69 \\
120 & 34.85 & 0.36 \\
150 & 29.48 & 0.35 \\
200 & 26.63 & 0.81 \\
250 & 23.87 & 0.88 \\
300 & 21.76 & 0.35 \\
400 & 19.07 & 0.68 \\
500 & 16.29 & 0.39 \\
600 & 14.67 & 0.72 \\
700 & 13.67 & 0.43 \\
800 & 12.69 & 0.23 \\
900 & 11.63 & 0.25 \\
1000 & 11.10 & 0.14 \\
\hline \hline
\end{tabular}

Mozejko et al. ${ }^{18}$ present two sets of TCS data: results from Gdansk for incident energies between 0.6 and $250 \mathrm{eV}$, and measurements from Trento for 90-3500 eV impact energies. Comparing our data with those for the lower energies, we found good agreement, to within the stated uncertainty limits, from 1 to $25 \mathrm{eV}$. Above $25 \mathrm{eV}$, their values tend to be lower in magnitude than ours, reaching maximum discrepancies of about $30 \%$ at around $200 \mathrm{eV}$. The energy resolution of the Gdansk apparatus is good enough to avoid contamination from most inelastic channels, but this is not probably the case in respect to their angular resolution. The contribution of electrons elastically scattered into the acceptance angle $(0.7 \mathrm{msr})$ of their detector ${ }^{18}$ is not discussed in Ref. 18, but it could lower their observed TCS values by about $15 \%-20 \%$ at $200 \mathrm{eV}$. As we discussed in previous publications ${ }^{42}$ (see Ref. 42 and references therein), this systematic error is really quite significant for a Ramsauer-type apparatus such as that used in Trento. ${ }^{18}$ Comparing the higher energy results from Trento with those of the present LTB system (with $<10^{-5}$ sr angular acceptance), we find that the former are about $42 \%$ lower in value than ours at $1000 \mathrm{eV}$. High resolution measurements using photoelectron (from synchrotron radiation) beams were carried out by Gulley et al., ${ }^{19}$ for impact energies below $2 \mathrm{eV}$. Their results, being generally higher in magnitude than the present ones, nonetheless agree with ours to within $15 \%$ even for the position and magnitude of the local peak maximum at around $1.4 \mathrm{eV}$. However, they found two other maxima at energies of around 1.17 and $1.29 \mathrm{eV}$ that are not present in our results. The origin of these resonances will be discussed in the next paragraph. Makochekanwa et al. ${ }^{21}$ and Kimura et al..$^{22}$ repeated the earlier measurements from Sueoka ${ }^{17}$ in order to 
TABLE III. Integral elastic cross sections $\left(10^{-20} \mathrm{~m}^{2}\right)$ as calculated with the SMCPP method, and total electron scattering cross sections $\left(10^{-20} \mathrm{~m}^{2}\right)$ as derived from the IAM-SCAR+I procedure for benzene.

\begin{tabular}{|c|c|c|c|}
\hline \multirow[b]{2}{*}{ Energy $(\mathrm{eV})$} & \multicolumn{2}{|c|}{ SMCPP (elastic integral cross section $\times 10^{-20} \mathrm{~m}^{2}$ ) } & \multirow{2}{*}{$\begin{array}{c}\text { IAM-SCAR }+\mathrm{I} \\
\left(\text { total cross section } \times 10^{-20} \mathrm{~m}^{2}\right)\end{array}$} \\
\hline & SEP1 & SEP2 & \\
\hline 0.1 & 101.1 & 101.1 & \\
\hline 0.2 & 44.65 & 44.66 & \\
\hline 0.3 & 24.67 & 24.62 & \\
\hline 0.4 & 15.81 & 15.64 & \\
\hline 0.5 & 11.52 & 11.20 & \\
\hline 0.6 & 9.554 & 9.063 & \\
\hline 0.7 & 8.937 & 8.274 & \\
\hline 0.8 & 9.169 & 8.339 & \\
\hline 0.9 & 9.956 & 8.869 & \\
\hline 1.0 & 11.10 & 9.960 & 107.8 \\
\hline 1.2 & 14.32 & & \\
\hline 1.3 & 18.7 & & \\
\hline 1.4 & 71.24 & & \\
\hline 1.5 & 22.21 & 17.86 & 87.92 \\
\hline 1.6 & 20.66 & 22.89 & \\
\hline 1.7 & 21.50 & 44.90 & \\
\hline 1.8 & 22.65 & 63.16 & \\
\hline 1.9 & 23.94 & 37.95 & \\
\hline 2 & 25.25 & 30.07 & \\
\hline 2.1 & 26.53 & 28.43 & 78.68 \\
\hline 2.2 & 27.75 & 28.38 & \\
\hline 2.3 & 28.91 & 28.86 & \\
\hline 2.4 & & 29.52 & \\
\hline 2.5 & 30.96 & 30.25 & \\
\hline 3 & 34.81 & 33.61 & \\
\hline 3.5 & 37.70 & 36.47 & 71.40 \\
\hline 4 & 39.43 & 38.33 & \\
\hline 4.3 & 41.33 & 40.35 & 67.20 \\
\hline 4.5 & 43.35 & 42.46 & \\
\hline 4.8 & 49.53 & 48.78 & \\
\hline 4.9 & 51.23 & 50.53 & \\
\hline 5 & 50.97 & 5032 & \\
\hline 5.3 & 46.85 & 46.46 & 63.56 \\
\hline 5.5 & 46.34 & 45.95 & \\
\hline 5.8 & 46.84 & 46.59 & \\
\hline 6 & 49.98 & 49.83 & \\
\hline 6.5 & 48.77 & 48.85 & \\
\hline 7 & 50.13 & 50.39 & \\
\hline 7.5 & 53.41 & 53.84 & 59.92 \\
\hline 8 & 51.83 & 52.38 & \\
\hline 8.5 & 52.42 & 53.06 & \\
\hline 9 & 51.19 & 51.90 & \\
\hline 9.5 & 52.51 & 53.28 & \\
\hline 10 & 52.55 & 53.37 & 57.12 \\
\hline 12 & 50.66 & 51.44 & \\
\hline 14 & 49.92 & 50.02 & \\
\hline 15 & 49.87 & 48.75 & 54.88 \\
\hline 20 & & & 53.76 \\
\hline 30 & & & 52.08 \\
\hline 40 & & & 49.56 \\
\hline
\end{tabular}


TABLE III. (Continued.)

\begin{tabular}{|c|c|c|c|}
\hline \multirow[b]{2}{*}{ Energy $(\mathrm{eV})$} & \multicolumn{2}{|c|}{ SMCPP (elastic integral cross section $\times 10^{-20} \mathrm{~m}^{2}$ ) } & \multirow{2}{*}{$\begin{array}{c}\text { IAM-SCAR }+\mathrm{I} \\
\left(\text { total cross section } \times 10^{-20} \mathrm{~m}^{2}\right)\end{array}$} \\
\hline & SEP1 & SEP2 & \\
\hline 50 & & & 46.76 \\
\hline 70 & & & 42.56 \\
\hline 100 & & & 37.8 \\
\hline 150 & & & 32.48 \\
\hline 200 & & & 28.28 \\
\hline 300 & & & 23.1 \\
\hline 400 & & & 19.54 \\
\hline 500 & & & 17.05 \\
\hline 700 & & & 13.61 \\
\hline 1000 & & & 10.53 \\
\hline 2000 & & & 6.05 \\
\hline 3000 & & & 4.26 \\
\hline 5000 & & & 2.68 \\
\hline 10000 & & & 1.38 \\
\hline
\end{tabular}

improve their accuracy. As shown in Fig. 3, those new results are in very good agreement with the present experimental data from 1 to $200 \mathrm{eV}$. Above this energy, however, their results tend to be lower than ours reaching a maximum discrepancy of about $17 \%$ at $1000 \mathrm{eV}$. This discrepancy seems to be related to the different angular resolutions of the respective experimental configurations and will be discussed later.

An interesting practical aspect in comparing experimental and theoretical TCS values is the possibility of identifying electron scattering resonances which have been theoretically predicted. The two approaches we used to describe the polarization (SEP1 and SEP2) in

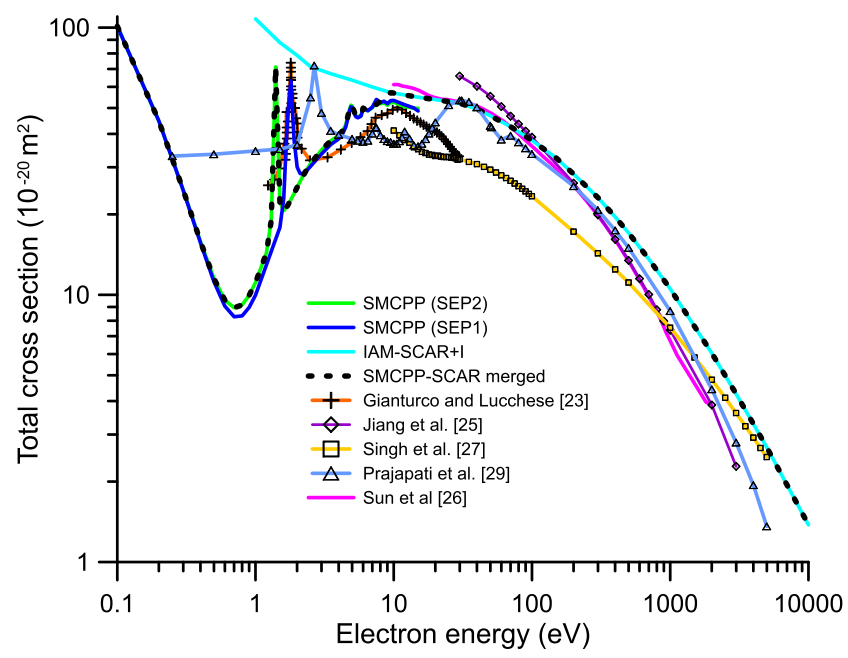

FIG. 2. Present TCS and elastic ICS calculations together with other theoretical values available in the literature (see also legend on the figure). our SMCPP method lead to a different representation (energy position and width) of the resonances (see Ref. 28 for details). In the energy range $1-10 \mathrm{eV}$, we can identify increments on the present measured total cross section around specific energy values (which we associate with the resonances). We found a first local maximum at $1.4 \pm 0.2 \mathrm{eV}$ that coincides with the energy of the ${ }^{2} \mathrm{E}_{2 \mathrm{u}}$ electronic state of the benzene anion ${ }^{43,44}$ and can be attributed to electron attachment to the $\pi^{*}$ virtual molecular orbital. ${ }^{19}$ This position is in excellent agreement with our SMCPP-SEP2 calculation, which placed a $\pi^{*}$ shape resonance at $1.4 \mathrm{eV}$ having a width of $0.05 \mathrm{eV}$. Note that this structure was also found by Mozejko et al. ${ }^{18}$ and Makochekanwa et al. ${ }^{21}$ Associated with the ${ }^{2} \mathrm{E}_{2 \mathrm{u}}$ resonance, Gulley et al. ${ }^{19}$ actually found three peaks in their TCS measurements. One was located at $1.4 \mathrm{eV}$, but the other two were at 1.29 and $1.17 \mathrm{eV}$,

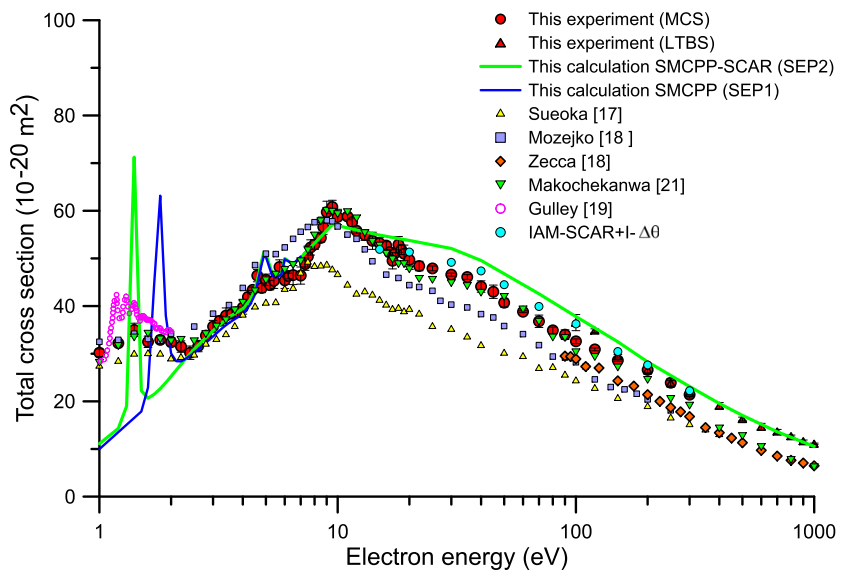

FIG. 3. Available experimental and present theoretical total cross sections for electron scattering from Benzene $\left(\mathrm{C}_{6} \mathrm{H}_{6}\right)$ (see also legend on the figure). 
respectively. There is no other experimental evidence for these two lower energy peaks, but we should note that the energy resolution of Gulley's experiment $(\sim 3.5 \mathrm{meV})$ allowed them to distinguish the vibronic components of this electronic resonance, while the other results give averaged cross sections over their respective energy resolutions (200 $\mathrm{meV}$ in the present case). Hence, there is no conflict between our observations and those of Gulley et al. ${ }^{19}$ Electron transmission experiments from Sanche and Schulz, ${ }^{45}$ Nenner and Schulz, ${ }^{46}$ and Burrow et al. ${ }^{47}$ did resolve the vibronic structure of this resonance, finding the first peak at $1.14,1.14$, and $1.12 \mathrm{eV}$, respectively. Calculations from Gianturco and Lucchese ${ }^{23}$ placed this resonance at $1.82 \mathrm{eV}$, in concordance with the $1.8 \mathrm{eV}$ position derived from our SMCPP-SEP1 results. The SMC calculations of Bettega et al. ${ }^{24}$ located this resonance at $2.3 \mathrm{eV}$. The recent calculation of Prajapati et al., ${ }^{29}$ using the R-matrix method with the Quantemol-N code, found the first peak of the TCS at $2.67 \mathrm{eV}$ in clear disagreement with all previous theoretical and experimental studies. This might reflect their use of a radius, typically $10 a_{0}$ in R-matrix applications, that is too small to accurately account for the anionic structure. Going to higher energies, we can distinguish a shoulder on the TCS energy dependence at around $3.4 \mathrm{eV}$, which is coincident with the local maximum found by Holst and Holtsmark ${ }^{16}$ at $3.5 \mathrm{eV}$. The origin of this peak is not clear, but we should note that similar structures were visible in the benzene derivatives ${ }^{5,48}$ pyridine and pyrimidine which were attributed to the vibrational excitation of the ground state (see Ref. 5 and references therein).

The next maximum we measured is located at $4.6 \pm 0.2 \mathrm{eV}$, confirming our calculated resonance for which both the SEP1 and SEP2 methods predict a position in the peak at $4.9 \mathrm{eV}$ with a width of $0.56 \mathrm{eV}$. This peak can be interpreted as a $\pi^{*}$ resonance $\left({ }^{2} \mathrm{~B}_{2 \mathrm{~g}}\right.$ symmetry) which is a mixture of a shape and a core excited resonance due to the presence of triplet excited states near the shape resonance energy. Li et al. ${ }^{49}$ computed, for the vertical excitation energy of the $1^{1} \mathrm{~B}_{2 \mathrm{u}}$ state, a value of $5.06 \mathrm{eV}$, which is in excellent agreement with the energy loss spectra recorded by Doering. ${ }^{50}$ Electron transmission experiments also found this resonance to be at $4.82 \mathrm{eV}^{47}$ and $5.1 \mathrm{eV} .{ }^{45}$ Due to the energy resolution limitations in the other available experiments, this resonance presents as a shoulder on the energy dependence of the TCS between 4.5 and $5 \mathrm{eV} .^{17,18,21}$ This feature is not noted in the calculations of Gianturco and Lucchese $^{23}$ and Prajapati et al., ${ }^{29}$ but they rather found resonances at 7.44 and $7.41 \mathrm{eV}$, respectively. Bettega et al. ${ }^{24}$ reported this resonance at $8.3 \mathrm{eV}$. Azria and Schulz ${ }^{51}$ suggested that this resonance could not only decay in the ground state ${ }^{1} \mathrm{~A}_{\mathrm{g}}$ but also in the ${ }^{3} \mathrm{~B}_{1 \mathrm{u}},{ }^{3} \mathrm{E}_{1 \mathrm{u}}$, and ${ }^{1} \mathrm{~B}_{1 \mathrm{u}}$ excited states. Allan ${ }^{52}$ reported that the ${ }^{2} \mathrm{~B}_{2 \mathrm{~g}}$ resonance has a relatively large importance in the excitation of the ${ }^{3} \mathrm{~B}_{1 \mathrm{u}},{ }^{3} \mathrm{E}_{1 \mathrm{u}}$ states, which confirms the interaction of a ${ }^{2} \mathrm{~B}_{2 \mathrm{~g}}$ shape-resonance with $\mathrm{a}^{2} \mathrm{~B}_{2 \mathrm{~g}}$ core-excited resonance. In addition, at $5.7 \pm 0.2 \mathrm{eV}$, we can distinguish a weak local maximum in our TCS values, which may correspond to the "Feshbach" resonance at $5.87 \mathrm{eV}$ proposed by Allan and associated with the $3 \mathrm{~s}\left(\mathrm{a}_{1 \mathrm{~g}}\right)$ Rydberg state. Between 6.5 and $8 \mathrm{eV}$, our experimental cross section increases more rapidly in magnitude, as a result of a superposition of electronic excited states. ${ }^{43,50-5}$ The absolute maximum value of the present TCS measurements, $(60.8 \pm 1.4) \times 10^{-20} \mathrm{~m}^{2}$, is reached at $9.5 \mathrm{eV}$, forming a broad structure around it. As the first ionization threshold in benzene is $9.2 \mathrm{eV},{ }^{53}$ and considering the high density of accessible states around this energy, our results do not have a good enough energy resolution in order to analyze the composition of such a broad structure. However, similar broad maxima within $8-10 \mathrm{eV}$ can be found in most representative experimental and theoretical studies. ${ }^{18,21,23}$ Allan $^{43}$ justified this broad maximum as being due to a ${ }^{2} E_{1 u}$ shape resonance produced by incident electron attachment to the $\sigma^{*}\left(\mathrm{e}_{1 \mathrm{u}}\right)$ orbital. Note that Prajapati et al. ${ }^{29}$ calculated the absolute maximum of the TCS at $30 \mathrm{eV}$, in disagreement with all the previous studies. Nonetheless note that our experimental TCSs do show a shoulder at around $30 \mathrm{eV}$ that may be coincident with the maximum in the sum over all the electronic-state excitation integral cross sections.

Concerning the higher energies, we can see from Figs. 2 and 3 some quite serious discrepancies between the energy dependencies given by the different measurements and calculations. However, this energy dependence is crucial for modeling radiation damage. Monte Carlo track simulations ${ }^{54}$ start from high energy (typically in the $\mathrm{MeV}$ range) electrons, which subsequently slowdown in the medium by successive collision processes until their final thermalization. ${ }^{55}$ Collisional data for energies above $10 \mathrm{keV}$ are customarily taken from atomic electron scattering cross section libraries (e.g., the LLNL Evaluated Electron Data Library ${ }^{56}$ ), which are based on the first Born approximation. ${ }^{13,57,58}$ To ensure consistency between the low-intermediate $\left(0-10^{3} \mathrm{eV}\right)$ and high-energy $\left(>10^{4} \mathrm{eV}\right)$ domains, the energy dependence of the TCS should smoothly overlap in the range $\left(10^{3}-10^{4} \mathrm{eV}\right)$. The high-energy dependence of the total cross section can easily be derived by assuming that at such high energies the molecules behave as a sum of atoms, with the energy dependence of the atomic total cross section $\left(\sigma_{\mathrm{T}}\right)$ being given by the Born formula, 57,58

$$
\sigma_{T}=\frac{A}{E}+\frac{B}{E} \ln E+\cdots,
$$

where $E$ represents the incident electron energy and $A$ and $B$ are constants related to the oscillator strength distribution of the target. ${ }^{57,58}$ Only first order terms are represented in Eq. (5), so it can be considered as the asymptotic energy dependence of the total cross section derived from the Born approximation. The first term in the above series represents the contribution of elastic scattering, while the second accounts for the inelastic processes (electronic excitation and ionization). Note that in a logarithmic plot both terms give straight lines whose slopes are -1 and -0.87 , respectively (see Fig. 4). In order to compare this energy dependence with that of the available TCS values, we have plotted where possible in Fig. 4 the theoretical and experimental data shown in Figs. 2 and 3, for energies above $500 \mathrm{eV}$, but normalizing to a value of 10 their respective results at $500 \mathrm{eV}$. As shown in Fig. 4, the previous calculations do not give asymptotic energy dependencies in agreement with the Born approximation. Assuming that Eq. (5) reasonably represents the energy dependence of the total cross section for electron energies above $1000 \mathrm{eV}$, we found that the results from both Jiang et al..$^{25}$ and Prajapati et al. ${ }^{29}$ lead to slopes higher than -1 . This is in clear contradiction with the $\mathrm{E}^{-1}$ energy dependence of the Born elastic cross section. In contrast, results from Singh et al..$^{27}$ give a slope lower than -0.87 and thus underestimate the $\mathrm{E}^{-0.87}$ energy dependence of the Born inelastic cross sections. However, as shown in Fig. 4, the corresponding slope from our IAM-SCAR+I method for energies above $1000 \mathrm{eV}$, is -0.92 , thereby giving an $\mathrm{E}^{-0.92}$ energy dependence for the TCS which is in good agreement with that predicted by the Born approximation. We can therefore fit our calculated TCSs from 1 to 


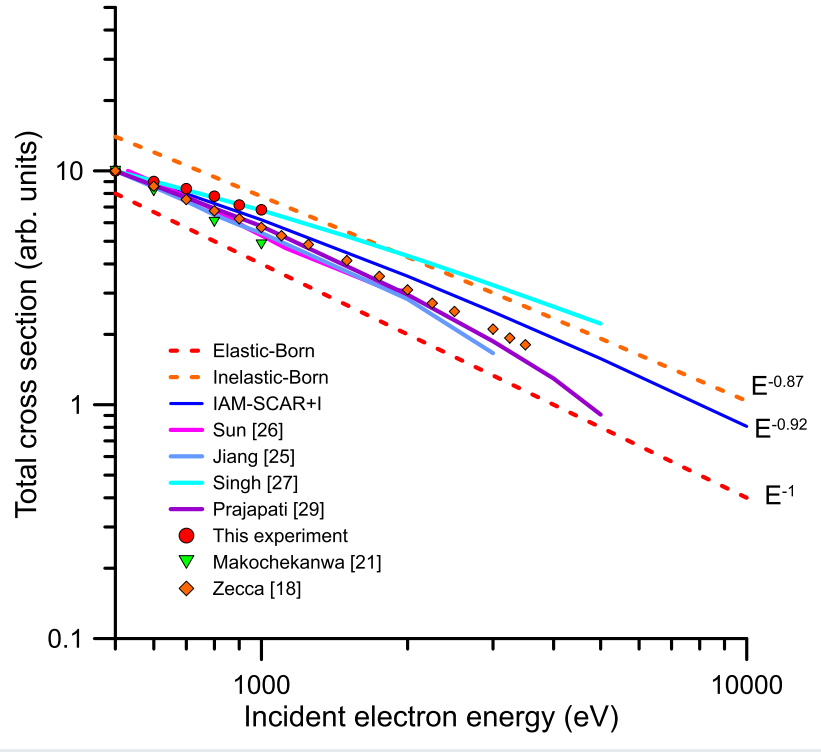

FIG. 4. Energy dependencies of the present and previous experimental and theoretical TCSs for energies above $500 \mathrm{eV}$.

$10(\mathrm{keV})$ to the expression given by Eq. (5) obtaining

$$
\sigma_{t}\left(10^{-20} \mathrm{~m}^{2}\right)=\frac{11.54}{E(k \mathrm{eV})}+\frac{1.035}{E(k \mathrm{eV})} \ln E(k \mathrm{eV})+\cdots
$$

This expression therefore allows us to extrapolate the TCS values for energies above $10 \mathrm{keV}$. Note that for very high energies, say above $100 \mathrm{keV}$, relativistic electron mass-velocity relationships should be included in Eq. (6) for a proper extrapolation.

\section{CONCLUSIONS}

In this study, we presented experimental results for the total electron scattering cross sections of benzene in the energy range $1-300 \mathrm{eV}$ by using a magnetically confined electron-transmission technique. Total random uncertainty limits on these values are within $5 \%$. The systematic error due to electrons elastically scattered into the acceptance angle of the detector (the so-called "missing angle" effect) has been estimated to be within 3\%-10\% depending on the incident energy. For the higher energies, from 100 to $1000 \mathrm{eV}$, additional linear electron beam-transmission measurements have been carried out with a superior angular resolution apparatus (acceptance angle $<10^{-5} \mathrm{sr}$ ). Random uncertainty limits on these results are within 5\%, with the systematic effect of the "missing angles" being negligible in this case. Good agreement, within the uncertainty limits, between both sets of our experimental data was found in the overlapping energy region $(100-300 \mathrm{eV})$. Reasonable agreement was also found with the most recent experimental data available in the literature ${ }^{18-21}$ for impact energies from 1 to $500 \mathrm{eV}$. Above this energy, however, the previous data are systematically lower in magnitude than the present results, probably due to the poorer angular resolution used in some of those measurements.
We have also presented the results of our calculations using two different methods, the SMCPP for impact energies from 0.1 to $15 \mathrm{eV}$ and the IAM-SCAR+I in the energy range $1-1000 \mathrm{eV}$. Due to the good agreement we found within their respective energy limits of applicability, we have merged both sets of data to obtain a full range (0.1-10000 eV) set of TCS values (labeled SMCPP-SCAR) with an estimated uncertainty limit of $\pm 10 \%$.

All the local maxima found in the low-energy dependence of our TCS have been identified as either shape or Feshbach resonances by comparing with previous theoretical and experimental studies and our SMCPP results. We can, in particular, highlight the $\pi^{*}$ shape resonance at $1.4 \mathrm{eV}$ and another feature in the energy region 4.6-4.9 eV, again predicted by our two SMCPP (SEP1 and SEP2) calculations and interpreted as a $\pi^{*}$ resonance $\left({ }^{2} \mathrm{~B}_{2 \mathrm{~g}}\right.$ symmetry) which is a mixture of shape and a core excited resonance. It was also noticeable that the Feshbach resonance at $5.87 \mathrm{eV}$, proposed by Allan ${ }^{52}$ and associated with the $3 \mathrm{~s}\left(\mathrm{a}_{1 \mathrm{~g}}\right)$ Rydberg state, appears as a shoulder in our TCS at around $5.7 \mathrm{eV}$.

Finally, the high-energy asymptotic behavior of our SMCPPSCAR calculated TCS values has been compared with the energy dependence predicted by the Born approximation. The good agreement found between both these theories allowed the derivation of an extrapolation formula to obtain TCS values for impact energies above $10 \mathrm{keV}$. We consider that this formula may be useful to link the present results with those of the existing electron data libraries, ${ }^{56}$ which are customarily used for modeling radiation damage.

\section{ACKNOWLEDGMENTS}

This study was partially supported by the Spanish Ministerio de Ciencia, Innovación y Universidades (Project No. FIS2016-80440) and by CSIC (Project No. LINKA20085). F.F.S. and P.L.V. acknowledge the Portuguese National Funding Agency FCT-MCTES [Research Grant Nos. UID/FIS/00068/2019 (CEFITEC), PTDC/FISAQM/31215/2017, and PTDC/FIS-AQM/31281/2017], while F.C. was supported by a Radiation Biology and Biophysics Doctoral Training Programme (RaBBiT) grant (RaBBiT, PD/00193/2012) and UID/Multi/04378/2013 479 (UCIBIO). Partial financial support from the Australian Research Council through Grant No. DP180101655 was also acknowledged. A.S.B. acknowledges support from Brazilian Agency Coordenação de Aperfeiçoamento de Pessoal de Nível Superior (CAPES). M.H.F.B. acknowledges support from Brazilian agency Conselho Nacional de Desenvolvimento Científico e Tecnológico (CNPq) and from FINEP (under project CTInfra). A.S.B. and M.H.F.B. also acknowledge computational support from Professor Carlos M. de Carvalho at LFTC-DFisUFPR and at LCPAD-UFPR and from CENAPAD-SP. We all thank Dr. L. Campbell for his help in preparing this paper.

\section{REFERENCES}

${ }^{1}$ Radiation Damage in Biomolecular Systems, edited by G. García Gómez-Tejedor and M. C. Fuss (Springer, London 2012).

${ }^{2}$ L. Sanche, Nature 461, 358 (2009).

${ }^{3}$ W. Chen, S. Chen, Y. Dong, P. Cloutier, Y. Zheng, and L. Sanche, Phys. Chem. Chem. Phys. 18, 32762 (2016).

${ }^{4}$ L. Ellis-Gibbings, A. D. Bass, P. Cloutier, G. García, and L. Sanche, Phys. Chem. Chem. Phys. 19, 13038 (2017). 
${ }^{5}$ A. I. Lozano, J. Jiménez, F. Blanco, and G. García, Phys. Rev. A 98, 012709 (2018).

${ }^{6}$ A. Traoré Dubuis, F. Costa, F. Ferreira da Silva, P. Limão-Vieira, J. C. Oller, F. Blanco, and G. García, Chem. Phys. Lett. 699, 182 (2018).

${ }^{7}$ A. I. Lozano, J. C. Oller, D. B. Jones, R. F. da Costa, M. T. do N. Varella, M. H. F. Bettega, F. Ferreira da Silva, P. Limão-Vieira, M. A. P. Lima, R. D. White, M. J. Brunger, F. Blanco, A. Muñoz, and G. García, Phys. Chem. Chem. Phys. 20, 22368 (2018).

${ }^{8}$ A. Loupas, A. I. Lozano, F. Blanco, J. D. Gorfinkiel, and G. García, J. Chem. Phys. 149, 034304 (2018).

${ }^{9}$ A. I. Lozano, A. Loupas, F. Blanco, J. D. Gorfinkiel, and G. García, J. Chem. Phys. 149, 134303 (2018).

${ }^{10}$ A. I. Lozano, F. Ferreira da Silva, F. Blanco, P. Limão-Vieira, and G. García, Chem. Phys. Lett. 706, 533 (2018).

${ }^{11}$ L. Feketeová, J. Postler, A. Zavras, P. Scheier, S. Denifl, and R. A. J. O’Hair, Phys. Chem. Chem. Phys. 17, 12598 (2015).

${ }^{12}$ K. Tanzer, L. Feketeová, B. Puschnigg, P. Scheier, E. Illenberger, and S. Denifl, J. Phys. Chem. A 119, 6668 (2015).

${ }^{13}$ H. Tanaka, M. J. Brunger, L. Campbell, H. Kato, M. Hoshino, and A. R. P. Rau, Rev. Mod. Phys. 88, 025004 (2016).

${ }^{14}$ M. J. Brunger, K. Ratnavelu, S. J. Buckman, D. B. Jones, A. Muñoz, F. Blanco, and G. García, Eur. Phys. J. D 70, 46 (2016).

${ }^{15}$ H. Kato, M. Hoshino, H. Tanaka, P. Limo-Vieira, O. Inglfsson, L. Campbell, and M. J. Brunger, J. Chem. Phys. 134, 134308 (2011).

${ }^{16}$ W. Holst and J. Holtsmark, Det. Kgl. Norske. Videndskab. Selskabs 4, 89 (1931).

${ }^{17}$ O. Sueoka, J. Phys. B: At., Mol. Opt. Phys. 21, L631 (1988).

${ }^{18}$ P. Możejko, G. Karsperski, Cz. Szmytkowski, G. P. Karwasz, R. S. Brusa, and A. Zecca, Chem. Phys. Lett. 257, 309 (1996).

${ }^{19}$ R. J. Gulley, S. Lunt, J. P. Ziesel, and D. Field, J. Phys. B: At., Mol. Opt. Phys. 31, 2735 (1998)

${ }^{20}$ G. P. Karwasz, R. S. Brusa, and A. Zecca, Rev. Nuovo Cim. 24, 1 (2001).

${ }^{21}$ C. Makochekanwa, O. Sueoka, and M. Kimura, Phys. Rev. A 68, 032707 (2003).

${ }^{22}$ M. Kimura, C. Makochekanwa, and O. Sueoka, J. Phys. B: At., Mol. Opt. Phys. 37, 1461 (2004).

${ }^{23}$ F. A. Gianturco and R. R. Lucchese, J. Chem. Phys. 108, 6144 (1998).

${ }^{24}$ M. H. F. Bettega, C. Winstead, and V. McKoy, J. Chem. Phys. 112, 8806 (2000).

${ }^{25}$ Y. Jiang, J. Sun, and L. Wan, Phys. Rev. A 62, 062712 (2000).

${ }^{26}$ J. Sun, C. Du, and Y. Liu, Phys. Lett. A 314, 150 (2003).

${ }^{27}$ S. Singh, R. Naghma, J. Kaur, and B. Antony, J. Chem. Phys. 145, 034309 (2016).

${ }^{28}$ A. S. Barbosa and M. H. F. Bettega, J. Chem. Phys. 146, 154302 (2017).

${ }^{29}$ D. Prajapati, H. Yadav, and P. C. Vinodkumar, Eur. Phys. J. D 72, 210 (2018).

${ }^{30}$ F. Blanco and G. García, Phys. Lett. A 317, 458 (2003).

${ }^{31}$ F. Blanco and G. García, Phys. Rev. A 67, 022701 (2003).

${ }^{32}$ F. Blanco, L. Ellis-Gibbings, and G. García, Chem. Phys. Lett. 645, 71 (2016).
${ }^{33}$ A. I. Lozano, J. C. Oller, K. Krupa, F. Ferreira Da Silva, P. Limão-Vieira, F. Blanco, A. Muñoz, R. Colmenares, and G. García, Rev. Sci. Instrum. 89, 063105 (2018).

${ }^{34}$ M. C. Fuss, A. G. Sanz, F. Blanco, J. C. Oller, P. Limão-Vieira, M. Brunger, and G. García, Phys. Rev. A 88, 042702 (2013).

${ }^{35}$ A. G. Sanz, M. C. Fuss, F. Blanco, J. D. Gorfinkiel, D. Almeida, F. Ferreira da Silva, P. Limão-Vieira, M. J. Brunger, and G. García, J. Chem. Phys. 139, 184310 (2013).

${ }^{36}$ A. Traoré Dubuis, A. Verkhovtsev, L. Ellis-Gibbings, K. Krupa, F. Blanco, D. B. Jones, M. J. Brunger, and G. García, J. Chem. Phys. 147, 054301 (2017).

${ }^{37}$ Y. Itikawa, J. Phys. Chem. Ref. Data 35, 31 (2006).

${ }^{38}$ R. F. da Costa, M. T. do N. Varella, M. H. F. Bettega, and M. A. P. Lima, Eur. Phys. J. D 69, 159 (2015).

${ }^{39}$ H. Kato, S. Kobayashi, C. Makochekanwa, M. Hoshino, N. Shinohara, O. Sueoka, H. Cho, and H. Tanaka, Phys. Rev. A 79, 062702 (2009).

${ }^{40} \mathrm{~J}$. Tennyson, Phys. Rep. 491, 29 (2010).

${ }^{41}$ See http://www.quantemol.com/ for information about available Quantemol software to calculate electron-molecule scattering cross sections.

${ }^{42}$ G. García, J. L. de Pablos, F. Blanco, and A. Williart, J. Phys. B: At., Mol. Opt. Phys. 35, 4657 (2002).

${ }^{43}$ M. Allan, J. Electron Spectros. Relat. Phenom. 48, 219 (1989).

${ }^{44}$ P. D. Burrow, J. A. Michejda, and K. D. Jordan, J. Chem. Phys. 86, 9 (1987).

${ }^{45}$ L. Sanche and G. J. Schulz, J. Chem. Phys. 58, 479 (1973).

${ }^{46}$ I. Nenner and G. J. Schulz, J. Chem. Phys. 62, 1747 (1975).

${ }^{47}$ P. D. Burrow, A. Modelli, and K. D. Jordan, Chem. Phys. Lett. 132, 441 (1986).

${ }^{48}$ A. I. Lozano, J. Jiménez, F. Blanco, and G. García, Phys. Rev. A 98, 012709 (2018).

${ }^{49}$ Y. Li, J. Wan, and X. Xu, J. Comput. Chem. 28, 1658 (2007).

${ }^{50}$ J. P. Doering, J. Chem. Phys. 51, 2866 (1969).

${ }^{51}$ R. Azria and G. J. Schulz, J. Chem. Phys. 62, 573 (1975).

${ }^{52}$ M. Allan, Helv. Chim. Acta 65, 2008 (1982).

${ }^{53}$ E. E. Koch and A. Otto, Chem. Phys. Lett. 12, 476 (1972).

${ }^{54}$ Radiation Damage in Biomolecular Systems, edited by A. Muñoz, M. C. Fuss, M. A. Cortés-Giraldo, S. Incerti, V. Ivanchenko, A. Ivanchenko, J. M. Quesada, F. Salvat, C. Champion and G. García in G. García Gómez-Tejedor, and M. C. Fuss (Canopus-Springer, London 2012).

${ }^{55}$ A. Muñoz, F. Blanco, G. Garcia, P. A. Thorn, M. J. Brunger, J. P. Sullivan, and S. J. Buckman, Int. J. Mass Spectrom. 277, 175-179 (2008).

${ }^{56}$ D. E. Cullen, J. H. Hubbell, and L. Kissel, EPDL97: The Evaluated Photon Data Library'97 Version, Lawrence National Laboratory, 1997, UCRL-LR-5400, Vol. 6, Rev. 5.

${ }^{57}$ M. Inokuti, Rev. Mod. Phys. 43, 297 (1971).

${ }^{58}$ M. Inokuti and M. R. C. McDowell, J. Phys. B 7, 2382 (1974). 